REVISTA DE ESTUDIOS E INVESTIGACIÓN EN PSICOLOGÍA Y EDUCACIÓN

\title{
Estrategias de lectura empleadas por estudiantes universitarios de tres áreas académicas
}

\section{Reading strategies used by undergraduate students from three academic areas}

\author{
Alejandra Platas-García (D) https://orcid.org/0000-0002-5150-0668 \\ Centro de Lenguas Extranjeras de la Facultad de Lenguas, Benemérita Universidad Autónoma \\ de Puebla: http://www.facultaddelenguas.com/cele \\ Puebla, Puebla - México \\ Verónica Reyes-Meza (iD https://orcid.org/0000-0002-2745-4032 \\ Centro Tlaxcala de Biología de la Conducta, Universidad Autónoma de Tlaxcala: \\ https://uatx.mx/facultad/CTBC \\ Tlaxcala, Tlaxcala - México \\ J. Martín Castro-Manzano (iD) https://orcid.org/0000-0003-2227-921X \\ Facultad de Filosofía, UPAEP (Universidad Popular Autónoma del Estado de Puebla): \\ https://www.upaep.mx/ \\ Puebla, Puebla - México
}




\title{
Resumen
}

Considerando la comprensión lectora como un proceso cognitivo en el que se relacionan características tanto del texto como del lector, en este trabajo se hizo la pregunta de si estudiantes universitarios pertenecientes a distintas áreas académicas emplean las mismas estrategias de lectura, a pesar de la diversidad de temáticas y de estructuras que poseen los textos académicos que leen. El objetivo del estudio fue, entonces, comparar la frecuencia de uso de las estrategias de lectura que reportan estudiantes universitarios, pertenecientes a tres áreas académicas, para conocer si existen o no diferencias entre ellos. Se realizó un estudio cuantitativo, comparativo, transversal, utilizando la traducción al español del Metacognitive Awareness of Reading Strategies Inventory. La muestra estuvo formada por 112 estudiantes de licenciatura pertenecientes a tres áreas académicas: Ciencias Sociales y Humanidades (52); Ingenierías y Ciencias Exactas (35); y Ciencias Naturales y de la Salud (25). Los resultados revelan que las estrategias de lectura de los participantes varían en función de su área académica.

Palabras clave: estrategias de lectura; metacognición; estudiantes universitarios de primer ciclo

\begin{abstract}
Considering reading comprehension as a cognitive process in which characteristics of both the text and the reader are related, in this paper was asked the question of whether university students from different academic areas employ the same reading strategies, in spite of the diversity of themes and structures that the academic texts read. Then, the aim of this study is to compare the frequency of use of the reading strategies reported by university students from three different academic areas, to assess the similarities and differences between them. A quantitative, comparative and cross sectional study was carried out using the Spanish translation of the Metacognitive Awareness of Reading Strategies Inventory. The sample consisted of 112 undergraduate students from three academic areas: Social Sciences and Humanities (52); Engineering and Exact Sciences (35); and Natural and Health Sciences (25). The findings reveal that the participants' reading strategies vary depending on their academic area.
\end{abstract}

Keywords: reading strategies; metacognition; undergraduate students 


\section{Estrategias de lectura}

La comprensión lectora vista desde una perspectiva interactiva es entendida como un proceso cognitivo en el que se relacionan características del texto y características del lector (cf. Kintsch y van Dijk, 1978). Así, en el contexto universitario, es conveniente considerar dos aspectos, a saber: los tipos de textos que leen los estudiantes y las estrategias de lectura que emplean; con respecto a esto, González (1988) asevera que los estudiantes universitarios al leer tienen que activar estrategias de lectura específicas para textos determinados con temáticas determinadas. Bajo este supuesto, surgió la pregunta de si estudiantes universitarios pertenecientes a distintas áreas académicas emplearían las mismas estrategias de lectura, a pesar de la diversidad de temáticas y de estructuras que poseen los textos académicos que suelen leer.

El objetivo fue, entonces, comparar la frecuencia de uso de las estrategias de lectura que reportan estudiantes universitarios, pertenecientes a tres áreas académicas, para conocer si existen o no diferencias entre ellos. Se realizó un estudio cuantitativo de corte transversal con el objetivo de comparar la frecuencia de uso de las estrategias de lectura que reportan estudiantes universitarios pertenecientes a tres áreas académicas. La hipótesis era que existen diferencias en el uso de las estrategias lectoras por parte de los grupos de estudiantes. Probablemente, las características de los textos que suelen leer los estudiantes en sus distintas áreas académicas inciden en el uso de estrategias lectoras que éstos emplean para cualquier tipo de texto académico y por ello, se pueden observar diferencias entre los grupos.

\section{Estrategias lectoras y su tipología}

Desde una perspectiva interactiva, se entiende por comprensión lectora, un proceso cognitivo a través del cual el lector construye un modelo de situación que le permite elaborar satisfactoriamente el significado del texto base con el cual interactúa a partir de los recuerdos presentes en su memoria (cf. van Dijk y Kintsch, 1983; van Dijk, 1993; Graesser et al., 1997; Pérez-Zorrilla, 2005).

Por su parte, Carrasco (2003) presenta una definición de comprensión lectora semejante a la anterior que destaca el rol que tienen las estrategias lectoras, a saber: "leer es un proceso de construcción de significados determinados culturalmente, durante el cual el lector, con los referentes y esquemas socialmente adquiridos, aplica estrategias diversas para construir una comprensión de un mensaje, comunicado a través de un texto escrito" (p. 131). En esta 
definición se advierte el rol mediático que tienen las estrategias lectoras, las cuales se pueden definir como "acciones aisladas o series de acciones que se realizan para lograr construir significado al leer un texto" (Carrasco, 2003, p. 132).

Se entiende por estrategias los "procedimientos utilizados para regular la actividad de las personas, en la medida en que su aplicación permite seleccionar, evaluar, persistir o abandonar determinadas acciones para llegar a conseguir la meta que nos proponemos" (Acosta, 2010). Además, existe un conjunto de nociones relacionadas con el concepto de estrategia; a saber: las estrategias son concebidas como habilidades, procedimientos, técnicas, métodos, algoritmos y heurísticos (Monereo, 1999).

Ahora bien, siguiendo la clasificación de Mokhtari y Reichard (2002), las estrategias lectoras pueden ser de tres tipos, a saber:

- Estrategias de lectura globales, se refieren a aquellas estrategias que son intencionales, son técnicas cuidadosamente planificadas por medio de las cuales los lectores monitorean o manejan su proceso de lectura.

- Estrategias de resolución de problemas, se refieren a las acciones y a los procedimientos que los lectores usan cuando trabajan directamente con el texto; es decir, corresponden a las técnicas utilizadas cuando se presentan problemas para comprender la información del texto.

- Estrategias de apoyo, se refieren a los mecanismos básicos de apoyo que tienen el objetivo de ayudar al lector en la comprensión del texto.

\section{Estrategias para leer textos especializados}

Los estudiantes universitarios pertenecientes a distintas áreas académicas enfrentan el problema de comprender textos especializados de su propia área de conocimiento; por ello, aprenden nuevas estrategias de lectura, las cuales son distintas de las que habían desarrollado para la comprensión de textos no especializados en los niveles educativos precedentes al nivel superior. Al respecto, Mateos (2009) menciona que cuando los estudiantes ingresan a la universidad se enfrentan a una nueva comunidad textual que tiene exigencias de lectura muy distintas a las que estaban habituados.

Así, además de utilizar conocimientos previos, realizar inferencias e identificar relaciones causales; ahora, también atienden a distintos aspectos como son: el contexto de producción del texto; los propósitos comunicativos y sociales; la interacción generada y los valores sociales presentes; la forma del texto y su variación en distintos contextos y tiempos (Castro y Sánchez, 
2016). Incluso, por esto, los estudiantes requieren una enseñanza que les permita comprender textos especializados, es decir, necesitan una "alfabetización académica" (Carlino, 2013).

Ahora bien, por un lado, desde una clasificación general de los textos en narrativos y expositivos; los textos especializados que leen los universitarios suelen ser expositivos. Este tipo de texto supone un mayor esfuerzo para los estudiantes en comparación con los textos narrativos (Ugarriza, 2006; Graesser et al. 2002). Por otro lado, desde una clasificación de los textos más particular para el contexto universitario, se puede decir que los estudiantes en ese nivel educativo se enfrentan a textos académicos que son explicativos-argumentativos (cf. Mateos, 2009). Los textos argumentativos tienen el propósito de demostrar o de refutar una tesis, parten de premisas para obtener una conclusión que puede ser una tesis nueva o una refutación de la tesis de partida (Álvarez-Angulo, 1997). Los textos explicativos-argumentativos que suelen leer los universitarios son textos científicos que requieren esfuerzo y concentración, ya que no suponen una lectura rápida (Graesser et al. 2002).

\section{Características de los textos especializados en tres áreas académicas}

Se presentan a continuación algunas particularidades que poseen los textos especializados que se emplean en las tres áreas académicas de interés para esta investigación, a saber: Ciencias Sociales y Humanidades; Ingenierías y Ciencias Exactas; y Ciencias Naturales y de la Salud.

Los textos en Ciencias Sociales y Humanidades se centran, comúnmente, en la discusión crítica de ideas e interpretaciones; los autores aprecian la variación estilística, por ello, suelen formular la misma idea de múltiples formas; los textos, además, proveen señales a los lectores de los puntos a los que deberían prestar especial atención, por ejemplo con frases o enunciados como: "una parte importante"; asimismo, varias discusiones en esta área comparan diferentes puntos de vista y mencionan la probabilidad de que alguno sea el mejor (Biber, 2005).

Los textos en Ingenierías y Ciencias Exactas suelen utilizar un discurso riguroso y preciso; se emplean frases y párrafos breves; existe una organización estándar; utilizan formas propias de comunicar el conocimiento como son los gráficos, los esquemas, los símbolos, o las expresiones matemáticas (cf. Espinoza, 2006). También, estos textos emplean, con frecuencia, expresiones que hacen referencia a las habilidades necesarias para llevar a cabo procesos o cálculos técnicos (casi siempre son construcciones pasivas): por ejemplo: "puede determinarse mediante", "pueden ser utilizadas para"; además, se emplean expresiones de tipo cuantitativo para indicar el estatus matemático de la frase nominal siguiente, por ejemplo: "a una aceleración de", "la raíz cuadrada de"; este tipo de expresiones cuantitativas se emplean también para 
identificar las relaciones entre los componentes y las variables utilizadas en ecuaciones matemáticas (Biber, 2005).

Los textos en Ciencias Naturales y de la Salud son difíciles en el sentido de que para acceder a un conocimiento del área, hace falta recurrir a otros conceptos con los que existe una relación cercana; por ejemplo, si se quiere abordar el concepto de materia, se necesita hacer referencia a las sustancias, la temperatura, el calor, los estados de agregación (sólido, líquido y gaseoso): cada uno de estos términos pueden tener una o varias acepciones en el lenguaje cotidiano y otra más rigurosa en el campo científico (Espinoza, 2006). Asimismo, estos textos transmiten contenido técnico denso, empleando términos y expresiones específicas para referirse a ese contenido, esto con el propósito de lograr una transmisión explícita de los significados; además, suelen emplear expresiones para identificar la localización física de algo, o alguna relación física con algo, como: "en la superficie de", "la posición de" (Biber, 2005).

\section{Conciencia de las estrategias lectoras}

En general, sobre el aprendizaje, se espera que los estudiantes universitarios construyan sus propios conocimientos adoptando un papel activo, autorregulado y que sean conocedores de sus propios procesos cognitivos (Hernández-Garre y Avilés-Soler, 2015).

En particular, sobre la lectura, se sabe que "el lector que controla su propia comprensión tiene una base óptima para elegir la mejor estrategia lectora que necesita en cada momento" (Jiménez et al., 2016, p. 288). En consecuencia, cuando un lector tiene conciencia de las estrategias que emplea al leer un texto, esto le ofrece grandes beneficios en el nivel educativo superior desde el punto de vista de su aprendizaje (cf. López-Delgado, 2002).

El control del propio proceso de comprensión forma parte de la metacognición, que consiste en la capacidad de planificar, supervisar, evaluar y modificar los procesos cognitivos, a fin de mejorar los resultados de la comprensión (García-García, 1993). Además, el conocimiento metacognitivo es de tipo condicional porque hace referencia "al qué, cómo, cuándo, dónde y en qué condiciones se deben utilizar ciertos recursos y estrategias para lograr aprendizajes o solucionar problemas" (Hernández, 1998, p. 143).

El componente metacognitivo desempeña un rol esencial en el proceso de la comprensión lectora, puesto que, gracias a éste, los estudiantes asumen "el control, la supervisión y la evaluación de su propio proceso de comprensión" (Cerchiaro et al., 2011, p. 106). Además, "el conocimiento metacognitivo permite al lector seleccionar, emplear, controlar y evaluar el uso de estrategias lectoras" (Pérez y González-Galli, 2020, p. 393). Por lo anterior, específicamente 
en contextos académicos, la metacognición de los universitarios favorece su proceso de comprensión de lectura (Valenzuela, 2018).

En suma, es probable que el tipo de textos especializados que tienen que enfrentar los estudiantes, debido al área académica a la que pertenecen, influya en las estrategias lectoras que utilizan de forma consciente.

\section{Método}

\section{Participantes}

Los participantes, seleccionados por conveniencia, fueron 112 estudiantes universitarios pertenecientes a dos universidades públicas del centro de México ( 80 mujeres y 32 hombres), con una edad promedio de 21.1 años (SD \pm 1.89 ). Los criterios de inclusión fueron: ser estudiantes que estuvieran cursando estudios de licenciatura en diferentes semestres y pertenecieran a una de las tres áreas académicas de interés en esta investigación. Si bien, las licenciaturas seleccionadas non son las únicas existentes en las universidades de los participantes dentro de cada área académica, se incluyeron estas por ser en las que se tuvo la oportunidad de aplicar el instrumento.

Los participantes se agruparon de la siguiente manera: Ciencias Sociales y Humanidades (52); Ingenierías y Ciencias Exactas (35); y Ciencias Naturales y de la Salud (25). Esta clasificación y el tipo de programas en que los estudiantes estaban inscritos se pueden visualizar en la Tabla 1 a continuación.

\section{Tabla 1}

Clasificación de los participantes por área académica

\begin{tabular}{lcl}
\hline Área académica & $n$ & Programas incluidos en las áreas \\
\hline Ciencias Sociales y Humanidades & 52 & Licenciatura en la Enseñanza del Inglés \\
Ingenierías y Ciencias Exactas & 35 & Ingeniería Química; Licenciatura en Matemáticas Aplicadas \\
Ciencias Naturales y de la Salud & 25 & Licenciatura en Biología; Licenciatura en Nutrición Humana \\
\hline
\end{tabular}

Nota: Esta división por área académica se tomó de la clasificación de las universidades de origen de los participantes.

\section{Instrumento}

Se empleó el cuestionario Metacognitive Awareness of Reading Strategies Inventory (MARSI) de Mokhtari y Reichard (2002) en la traducción al español realizada por Gómez et al. (2014). Este instrumento tiene como propósito medir el tipo y la frecuencia de uso de las estrategias de lectura que emplean los adultos mientras leen textos académicos. 
El cuestionario original cuenta con 30 enunciados y usa una escala de Likert con cinco opciones que indican la frecuencia de uso y que van de nunca (1) a siempre (5). Es necesario mencionar que, además de la traducción realizada, Gómez et al. (2014) agregaron un enunciado que hace alusión a la lectura en inglés como lengua extranjera. Sin embargo, dado que el interés de la presente investigación se centraba en la lectura en lengua materna, se optó por dejar el MARSI original con 30 enunciados.

Los autores Mokhtari y Reichard (2002) clasifican los enunciados en tres categorías: estrategias globales (13 enunciados), estrategias de resolución de problemas (8) y estrategias de apoyo (9). La puntuación total es el promedio de las puntuaciones de todos los enunciados. Este promedio permite clasificar a los estudiantes en tres niveles: nivel alto (de 3.5 a 5.00 puntos), nivel medio (de 2.5 a 3.4 puntos) y nivel bajo (de 0 a 2.4 puntos). Estos niveles indican la frecuencia de uso de las estrategias en general (referidas en los 30 enunciados). La aplicación tiene una duración máxima de 10 a 12 minutos.

\section{Procedimiento}

Distintos profesores universitarios solicitaron a sus estudiantes la participación voluntaria en esta investigación, a quienes aceptaron, se les informó que sus datos personales se usarían únicamente para los fines del estudio y que se reportarían de forma anónima. Posteriormente, les aplicaron el instrumento MARSI dentro de sus salones de clases habituales, se emplearon copias del cuestionario en hojas de papel. La duración fue de 10 a 12 minutos.

Para el análisis estadístico, se evaluó la normalidad de la distribución de los datos obtenidos mediante la prueba Shapiro-Wilk y, como los datos no pasaron la prueba de normalidad, se empleó la prueba de Kruskal-Wallis, la cual es una alternativa no paramétrica al ANOVA que se emplea para comparar las medianas obtenidas en tres o más grupos. Para realizar esta prueba se utilizó el software GraphPad Prism, 5.01 (GraphPad Software Inc., San Diego, USA).

\section{Resultados}

Para conocer si los estudiantes universitarios, pertenecientes a tres áreas académicas, emplean con la misma frecuencia las estrategias de lectura, se compararon sus respuestas con respecto a cada uno de los 30 enunciados del MARSI. Se empleó la prueba de Kruskal-Wallis y post-hoc de Dunn. Únicamente se presentan, a continuación, los resultados con diferencias estadísticamente significativas usando la clasificación de estrategias: globales, de resolución de problemas y de apoyo. 
A partir de aquí, con fines de facilitar la lectura, cuando se hará referencia a las tres áreas académicas se usarán las siguientes siglas en el texto, a saber: Ingenierías y Cs. Exactas (ICE); Cs. Sociales y Humanidades (CSH); Cs. Naturales y de la Salud (CNS).

\section{Estrategias de lectura globales}

Los enunciados que corresponden a las estrategias globales son: 1, 3, 4, 7, 10, 14, 17, 19, $22,23,25,26,29$. Cuando se compararon las respuestas de los estudiantes en cada una de las tres áreas académicas se encontraron diferencias significativas en los siguientes:

Enunciado 1: "Cuando leo tengo un objetivo/propósito en mente" $(k=6.733, n=112, p=$ 0.034). La prueba post-hoc de Dunn indicó que las diferencias se presentan entre las áreas de CSH e ICE. Así, haciendo un análisis de los porcentajes de frecuencia de uso se encontró que en ICE los estudiantes tienen conciencia de usar la estrategia 1 con más frecuencia que en CSH (ver Figura 1).

\section{Figura 1}

Porcentajes de respuestas para la estrategia 1

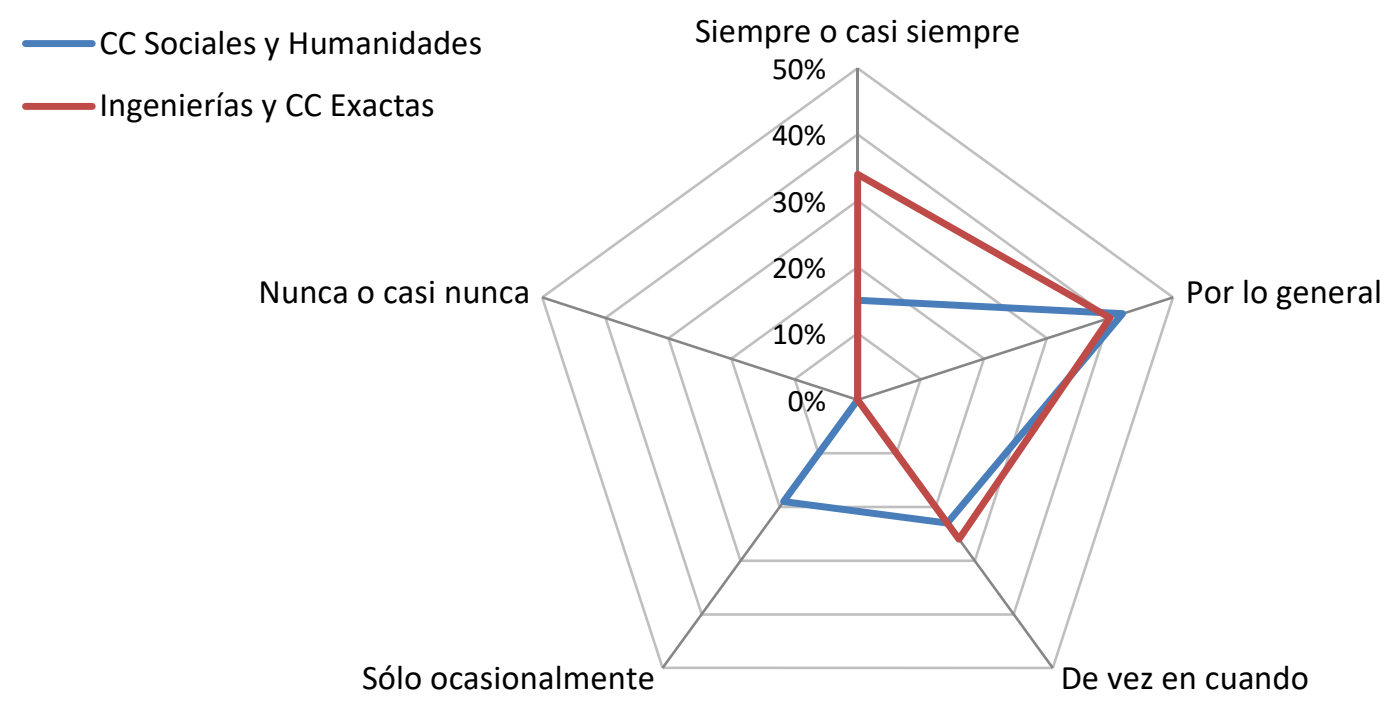

Enunciado 10: "Primero realizo una lectura rápida del texto para fijarme en sus características, como la longitud y la organización" $(k=7.934, n=112, p=.018)$. La prueba posthoc de Dunn indicó que las diferencias se presentan entre las áreas de CSH y CNS. Así, haciendo un análisis de los porcentajes de frecuencia de uso se encontró que en CNS los estudiantes tienen conciencia de usar la estrategia 10 con más frecuencia que los estudiantes de CSH (ver Figura 2). 


\section{Figura 2}

Porcentajes de respuestas para la estrategia 10

CC Sociales y Humanidades

Siempre o casi siempre

CC Naturales y de la Salud

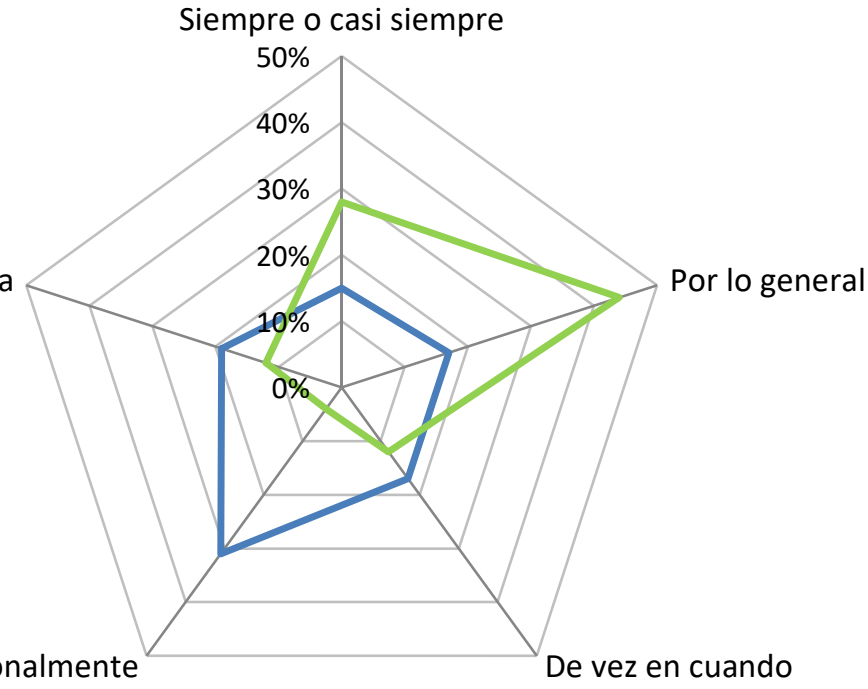

Sólo ocasionalmente

De vez en cuando

Enunciado 22: "Utilizo ayudas o recursos tipográficos como la negrita y la cursiva para identificar la información clave/importante" $(k=10.06, n=112, p=.006)$. La prueba post-hoc de Dunn indicó que las diferencias se presentan entre las áreas de ICE y CSH; asimismo, entre ICE y CNS. Así, haciendo un análisis de los porcentajes de frecuencia de uso se encontró que, en CSH y en CNS, los estudiantes tienen conciencia de usar con más frecuencia la estrategia 22 que en ICE (ver Figura 3).

\section{Figura 3}

Porcentajes de respuestas para la estrategia 22

CC Sociales y Humanidades

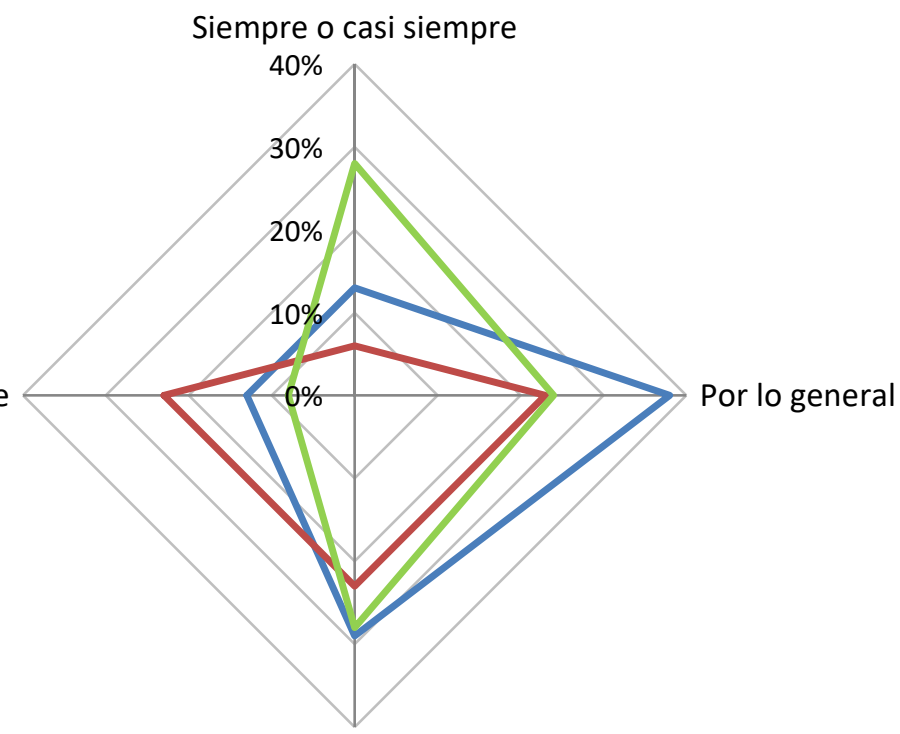

De vez en cuando 


\section{Estrategias de resolución de problemas en la lectura}

Los enunciados que corresponden a las estrategias de resolución de problemas son: 8, 11, $13,16,18,21,27,30$. Cuando se compararon las respuestas de los estudiantes en cada una de las tres áreas académicas se encontraron diferencias significativas únicamente en el siguiente:

Enunciado 11: "Trato de retomar el hilo cuando pierdo la concentración" ( $k=6.156, n=$ $112, p=.046)$. La prueba post-hoc de Dunn indicó que las diferencias se presentan entre las áreas de CNS e ICE. Así, haciendo un análisis de los porcentajes de frecuencia de uso se encontró que en CNS, los estudiantes tienen conciencia de usar la estrategia 11 con más frecuencia que en ICE (ver Figura 4).

\section{Figura 4}

Porcentajes de respuestas para la estrategia 11

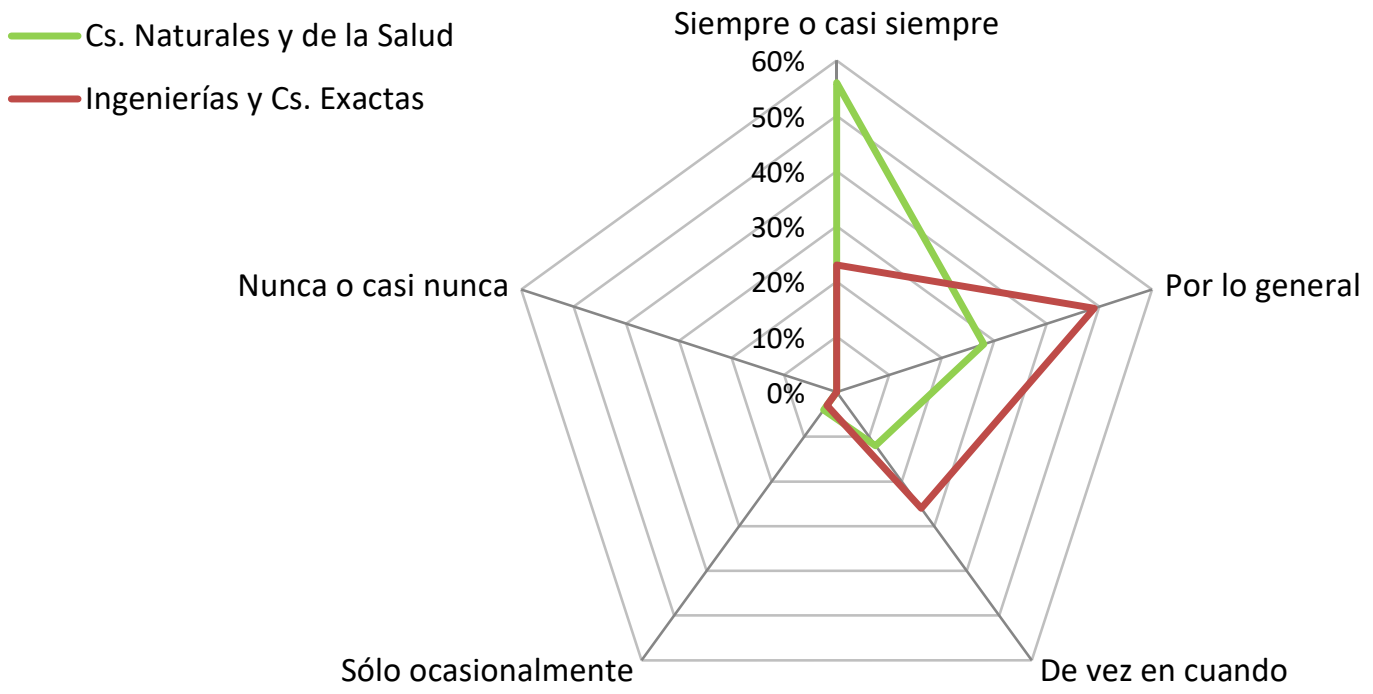

\section{Estrategias de apoyo para la lectura}

Los enunciados que corresponden a las estrategias de apoyo son: 2, 5, 6, 9, 12,15, 20, 24, 28. Cuando se compararon las respuestas de los estudiantes en cada una de las tres áreas académicas se encontraron diferencias significativas en los siguientes:

Enunciado 12: "Subrayo o rodeo información en el texto para ayudarme a recordarla" ( $k$ $=11.89, n=112, p=.002$ ). La prueba post-hoc de Dunn indicó que las diferencias se presentan entre las áreas de ICE y CSH; asimismo, entre ICE y CNS. Así, haciendo un análisis de los porcentajes de frecuencia de uso se encontró que en CSH y en CNS, los estudiantes tienen conciencia de usar la estrategia 12 con más frecuencia que en ICE (ver Figura 5). 


\section{Figura 5}

Porcentajes de respuestas para la estrategia 12

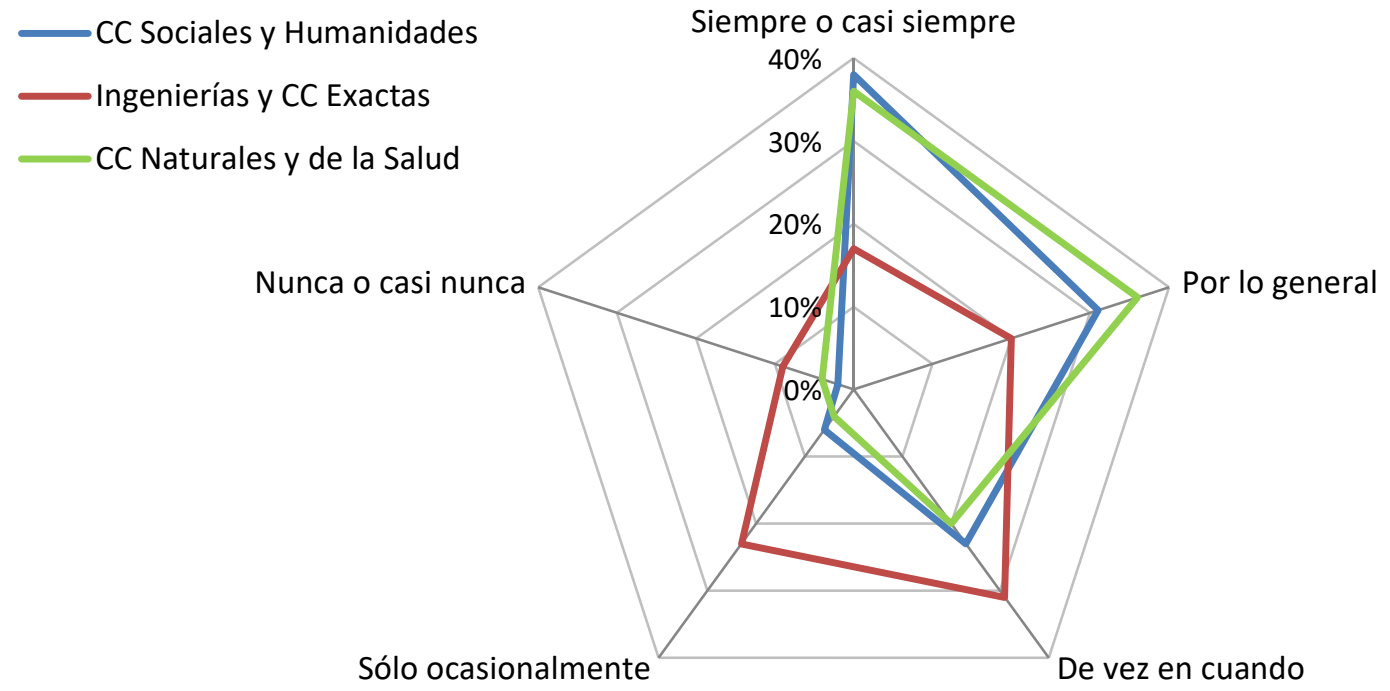

Enunciado 24: "Voy hacia atrás y hacia delante en el texto para encontrar en él relaciones entre las ideas" ( $k=7.848, n=112, p=.019$ ). La prueba post-hoc de Dunn indicó que las diferencias se presentan entre las áreas de CSH y CNS. Así, haciendo un análisis de los porcentajes de frecuencia de uso se encontró que en CNS los estudiantes tienen conciencia de usar la estrategia 24 con más frecuencia que en CSH (ver Figura 6).

\section{Figura 6}

Porcentajes de respuestas para la estrategia 24

CC Sociales y Humanidades

Siempre o casi siempre

CC Naturales y de la Salud

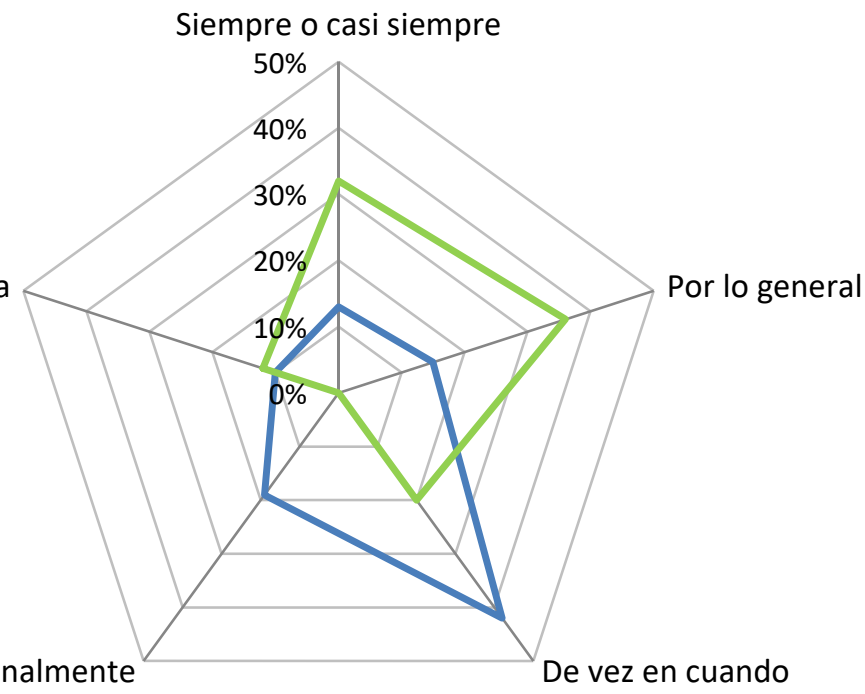

Sólo ocasionalmente

De vez en cuando 
En la Tabla 2 se presenta un resumen de los resultados anteriores.

\section{Tabla 2}

Diferencias estadísticamente significativas $(p<.05)$ entre áreas académicas para estrategias de lectura con mayor frecuencia de uso

\begin{tabular}{lcc}
\hline & \multicolumn{2}{c}{ Áreas académicas } \\
\cline { 2 - 3 } & $\begin{array}{c}\text { Ciencias Sociales y Humanidades } \\
\text { (CSH }\end{array}$ & $\begin{array}{c}\text { Ingenierías y Ciencias Exactas } \\
\text { (ICE) }\end{array}$ \\
\hline $\begin{array}{l}\text { Ciencias Naturales y y } \\
\text { de la Salud (CNS) }\end{array}$ & $\begin{array}{c}\text { Global: realizar una lectura rápida } \\
\text { para conocer la organización del } \\
\text { texto (estrategia 10). }\end{array}$ & $\begin{array}{c}\text { Global: ayudarse de recursos tipográficos } \\
\text { para identificar información importante } \\
\text { (estrategia 22). }\end{array}$ \\
& $\begin{array}{c}\text { Apoyo: encontrar relaciones entre las } \\
\text { ideas revisando el texto de arriba } \\
\text { abajo (estrategia 24). }\end{array}$ & $\begin{array}{c}\text { Apoyo: subrayar información para } \\
\text { recordarla (estrategia 12). }\end{array}$ \\
& $\begin{array}{c}\text { Resolución de problemas: buscar retomar } \\
\text { el hilo cuando se pierde la concentración } \\
\text { en la lectura (estrategia 11). }\end{array}$ \\
\hline $\begin{array}{l}\text { Ciencias Sociales y } \\
\text { Humanidades (CSH) }\end{array}$ & $\begin{array}{c}\text { Global: ayudarse de recursos tipográficos } \\
\text { para identificar información importante } \\
\text { (estrategia 22). }\end{array}$ \\
& & $\begin{array}{c}\text { Apoyo: subrayar información para } \\
\text { recordarla (estrategia 12). }\end{array}$ \\
\hline $\begin{array}{l}\text { Ingenierías y Ciencias } \\
\text { Exactas (ICE) }\end{array}$ & $\begin{array}{c}\text { Global: tener un propósito para la } \\
\text { lectura (estrategia 1). }\end{array}$ & \\
\hline
\end{tabular}

Finalmente, parece oportuno indicar que, por el promedio de puntuaciones en el MARSI, se ubicaron en el nivel alto primero los estudiantes de CNS con el 56\%, seguidos por los estudiantes de ICE con el 51\%, y finalmente por los estudiantes de CSH con el $46 \%$.

\section{Discusión}

Se discuten a continuación los resultados comparados en las áreas académicas en que hubo diferencias significativas.

\section{Ayudarse de recursos tipográficos y subrayar información (CSH y CNS vs ICE)}

La estrategia global de ayudarse de recursos tipográficos para identificar información importante (estrategia 22) es empleada con frecuencia por estudiantes de CSH y CNS; pero, es menos utilizada por estudiantes de ICE. De forma similar al caso anterior, la estrategia de apoyo que consiste en subrayar información importante para recordarla (estrategia 12) es empleada con frecuencia por estudiantes de CSH y CNS; pero es menos utilizada por estudiantes de ICE.

En relación con estos resultados, se puede mencionar el estudio que realizó Peretz (1986), en el cual investigó a estudiantes de tres áreas académicas (Ciencia y Tecnología; Humanidades; y Biología). Sus hallazgos muestran que los estudiantes de Ciencias y Tecnología (que en la 
presente investigación se podrían relacionar con los estudiantes de ICE) tuvieron un desempeño más alto en la prueba; menciona que esto podría deberse a las estrategias de comprensión lectora específicas que este grupo de estudiantes desarrolla, a saber: a los estudiantes de Ciencia y Tecnología se les enseña cómo comprender procesos, hipótesis, teorías y experimentos; mientras que los estudiantes de CSH suelen leer con el fin de determinar la idea principal del autor, las ideas que apoyan esa idea, y un punto de vista general (Peretz, 1986, p. 14).

Así, relacionando los resultados de Peretz (1986) con los de la presente investigación, parece que los estudiantes de ICE no necesitarían usar tan frecuentemente estrategias que les ayuden a encontrar ideas principales o secundarias (como serían ayudarse de recursos tipográficos -negritas, cursivas- o subrayar información importante) porque su objetivo de lectura es comprender procesos, hipótesis, teorías y experimentos, para lo cual no basta con resaltar palabras. Con esto no se quiere decir que las estrategias de ayudarse de recursos tipográficos y subrayar información importante no les sean útiles en ningún momento a los estudiantes de ICE; más bien, parece que podría explicar por qué en esta investigación emplearon estas estrategias con menos frecuencia (para un estudio sobre algunas estrategias cognitivas y metacognitivas que usan estudiantes de ciencias en la comprensión de un texto de ciencias, cf. Maturano et al., 2002).

\section{Mantener la concentración (CNS vs ICE)}

Los estudiantes de CNS reportan que usan con frecuencia la estrategia de retomar el hilo cuando pierden la concentración (estrategia 11), mientras que los estudiantes de ICE reportan que la emplean menos.

Probablemente, este resultado se podría explicar, en parte, debido a las características propias de los textos de cada una de las áreas académicas. Es decir, probablemente, los textos de CNS, al ser difíciles (Espinoza, 2006), hacen que los estudiantes se distraigan más fácilmente (y por ello, tengan que retomar el hilo), a diferencia de los textos de ICE. Con respecto a esto, por ejemplo, sobre los textos de matemáticas, Brennan y Dunlap (como se citó en Barton y Heidema, 2002) explican que estos textos contienen más conceptos por palabra, por enunciado y por párrafo que cualquier otro tipo de texto; adicionalmente, estos conceptos suelen ser abstractos lo que les dificulta a los lectores de otras áreas visualizar sus significados; asimismo, los autores de textos de matemáticas escriben generalmente con un estilo muy concreto, de tal forma que cada enunciado contiene información abundante y un poco redundante; además, los enunciados y las palabras a menudo tienen significados precisos y se conectan lógicamente con las oraciones circundantes. Todo esto hace que si un estudiante quisiera leer textos de 
matemáticas rápidamente (como lo podría hacer si tuviera que leer una historia corta en una clase de literatura), supondría perderse de detalles significativos, de explicaciones y de la lógica subyacente (Barton y Heidema, 2002).

Relacionando la referencia de Barton y Heidema (2002) con los resultados de la presente investigación, se puede decir que si los estudiantes de ICE no suelen usar tan frecuentemente la estrategia de mantener la concentración como lo hacen los estudiantes de CNS, podría deberse a que los estudiantes están habituados a realizar un esfuerzo ininterrumpido para poner atención al texto que están leyendo con el propósito de no perder detalles.

\section{Realizar una lectura rápida y revisar el texto de arriba a abajo (CNS vs CSH)}

Los estudiantes de CNS suelen usar frecuentemente la estrategia global de realizar una lectura rápida del texto para fijarse en sus características, como la longitud y la organización (estrategia 10), a diferencia de los estudiantes de CSH que la emplean menos. Asimismo, los estudiantes de CNS suelen usar con frecuencia la estrategia de apoyo de encontrar relaciones entre las ideas revisando el texto de arriba abajo (estrategia 24), a diferencia de los estudiantes de CSH que la emplean menos.

Una observación inicial sobre estos resultados está relacionada con el hecho de que el mayor porcentaje de estudiantes que se encontraban en el nivel alto del MARSI pertenecían al área de CNS; es decir, parece que en esta área académica los estudiantes emplean con más frecuencia estrategias de lectura de forma consciente, a comparación de las otras dos áreas.

Estos resultados se podrían explicar debido a las exigencias que los cursos en CNS suponen para los estudiantes. Por ejemplo, Momsen et al. (2010) mencionan que los cursos introductorios de la licenciatura en Biología dan excesiva importancia a la memorización de hechos y detalles; además, explican que en esta área se sigue la metodología cuantitativa y los estudiantes deben integrar conocimientos a través de escalas de tiempo y espacio. Estas características de los cursos permiten comprender por qué al leer un texto de su área académica, los estudiantes de CNS suelen realizar una lectura rápida para fijarse en detalles como su organización y longitud; asimismo, revisan el texto para encontrar relaciones, esto probablemente, debido a su enfoque cuantitativo.

Ahora bien, del lado de los estudiantes de CSH, parece que podrían verse favorecidos por el uso más frecuente de las estrategias de "la realización de una lectura rápida” y “la revisión del texto de arriba abajo". Por ejemplo, Gordillo y Flórez (2009) reportan que estudiantes de la licenciatura en Lengua Castellana, Inglés, y Francés, dependen de un nivel de lectura literal (por ejemplo, en búsqueda de una determinada palabra), hecho que podría verse relacionado con la realización de una lectura rápida. 


\section{Tener un propósito en mente (ICE vs. CSH)}

Los estudiantes de ICE suelen usar con frecuencia la estrategia global de tener un objetivo o propósito en mente cuando leen (estrategia 1), a diferencia de los estudiantes de CSH.

Del lado de los estudiantes de ICE, este resultado es consistente, por ejemplo, con los hallazgos de Bazerman (como se citó en López-Bonilla, 2017), quien analizó lo que hacen los físicos cuando leen textos de física y encontró que "todos los participantes establecían un propósito al leer que hacía de su lectura un proceso más selectivo, ya que ponían atención a ciertos elementos y no a otros, según sus necesidades de investigación" (p. 32).

Del lado de los estudiantes de CSH, tal vez, al ser su propósito de lectura más general, en el sentido de: determinar la idea principal del autor, las ideas que apoyan esa idea, y un punto de vista general (Peretz, 1986, p. 14); parece que este propósito no responde (como en el caso de los físicos) a necesidades específicas para acercarse a leer un texto. Necesidades específicas podrían ser, por ejemplo: encontrar argumentos a favor de un determinado tema de interés o conocer el diseño metodológico de una investigación.

Pues bien, a partir de la evidencia que se ha presentado, se puede concluir que, si bien perecería que todas las estrategias de lecturas consideradas en esta investigación (que conforman el cuestionario MARSI) son útiles, no todas se usan con la misma frecuencia por estudiantes de las tres áreas académicas consideradas. Se ha discutido que esto puede deberse, por ejemplo, a las características de los textos que leen los estudiantes o a las exigencias solicitadas en sus cursos.

Así, se puede sugerir una propuesta didáctica para promover la conciencia de las estrategias lectoras en las distintas áreas académicas, a saber: momentos en las clases universitarias dedicados a la lectura de textos académicos propios de cada disciplina, con el propósito de reflexionar en las estrategias lectoras que posibilitan la comprensión. Los docentes guiarían y acompañarían a los estudiantes en esos momentos dedicados a la lectura, reflexionando sobre las propias prácticas lectoras y las dificultades experimentadas, así como sobre los distintos tipos de textos que tienen que leer (Mateos, 2009). Asimismo, en este acompañamiento, sería oportuno analizar en qué medida las estrategias que suelen emplear los estudiantes universitarios pueden ser mejoradas (Maturano et al., 2002); esto con el propósito de guiar a los estudiantes para mejorar su proceso de comprensión lectora.

Es necesario reconocer una limitación de este estudio: se han considerado áreas académicas que abarcan un gran número de programas de licenciatura, sin embargo, se han estudiado únicamente algunos. Se piensa realizar un nuevo estudio con una muestra de 
participantes más amplia. Se podría llevar a cabo una investigación similar, pero complementando el MARSI con una prueba que midiera las estrategias lectoras empleadas por los estudiantes al leer un determinado texto académico propio de cada una de las disciplinas de interés. Así, por un lado, se conocería cuál es la frecuencia de uso de las estrategias lectoras desde el punto del auto-reporte de los estudiantes; $y$, por otro lado, se podría solicitar una tarea que permitiera observar el empleo de las estrategias lectoras de los estudiantes en su práctica.

La aportación de esta investigación ha consistido en abordar el auto-reporte sobre el uso de las estrategias lectoras de los estudiantes clasificados conforme a su área académica.

Parece que el análisis comparativo entre áreas académicas sobre las estrategias de lectura podría ser útil para evitar que las distintas áreas académicas se encerraran en su propio contexto y en sus prácticas de lectura de textos, sin detenerse a observar cómo ciertas estrategias de lectura han sido útiles para estudiantes e investigadores en otros contextos académicos.

\section{Referencias}

ACOSTA, lleana (2010). La comprensión lectora, enfoques y estrategias utilizadas durante el proceso de aprendizaje del idioma español como segunda lengua. [Tesis de doctorado, Universidad de Granada]. Repositorio Institucional de la Universidad de Granada. https://digibug.ugr.es/handle/10481/4969

ÁLVAREZ-ANGULO, Teodoro (1997). El texto argumentativo en Primaria y Secundaria. Didáctica. Lengua y Literatura, 9, 23-37. https://revistas.ucm.es/index.php/DIDA/article/view/DIDA 9797110023A

BARTON, Mary Lee; \& HEIDEMA, Clare (2002). Teaching reading in mathematics A Supplement to Teaching Reading in the Content Areas: If Not Me, Then Who? (2a. ed.). Mid-Continent Research for Education and Learning.

BIBER, Douglas (2005). Paquetes léxicos en textos de estudio universitario: Variación entre disciplinas académicas. Revista signos, 38(57), 19-29. https://doi.org/10.4067/S071809342005000100002

CARLINO, Paula (2013). Alfabetización Académica diez años después. Revista Mexicana de Investigación Educativa, 18(57), 355-381. http://comie.org.mx/revista/v2018/rmie/ index.php/nrmie/article/view/250

CARRASCO, Alma (2003). La escuela puede enseñar estrategias de lectura y promover su regular empleo. Revista Mexicana de Investigación Educativa, 8(17), 129-142. https://www.comie.org.mx/revista/v2018/rmie/index.php/nrmie/article/view/899 
CASTRO, María Cristina; \& SÁNCHEZ, Martín (2016). Características genéricas y estrategias de lectura. Una propuesta para la comprensión de textos académicos. En Gerardo Bañales; Montserrat Castelló; \& Norma Alicia Vega (Coords.), Enseñar a leer y escribir en la educación superior. Propuestas educativas basadas en la investigación (pp. 78-100). IDEA.

CERCHIARO, Elda; PABA, Carmelina; \& SÁNCHEZ, Ligia (2011). Metacognición y Comprensión Lectora: una relación posible e intencional. DUAZARY. Revista de la Facultad de Ciencias de la Salud, 8(1), 99-111. https://doi.org/10.21676/2389783X.258

ESPINOZA, Ana (2006). La especificidad de las situaciones de lectura en "Naturales". Lectura y Vida, 27(1), 6-16. http://www.lecturayvida.fahce.unlp.edu.ar/numeros/a27n1/sumario

GARCÍA-GARCÍA, Emilio (1993). La comprensión de textos. Modelo de procesamiento y estrategias de mejora. Didáctica. Lengua y Literatura, 5, 87-113. https://revistas.ucm.es/ index.php/DIDA/article/view/DIDA9393110087A

GÓMEZ, Ángela; SOLAZ, Joan Josep; \& SANJOSÉ, Vicente (2014). Competencia en lengua inglesa de estudiantes universitarios españoles en el contexto del EEES: Nivel de dominio lingüístico, estrategias metacognitivas y hábitos lectores. Revista de Educación, 363, 154183. https://doi.org/10.4438/1988-592X-RE-2012-363-175

GONZÁLEZ, Raúl (1998). Comprensión lectora en estudiantes universitarios iniciales. Persona: Revista de la Facultad de Psicología, 1, 43-65. https://doi.org/10.26439/persona1998. n001.691

GORDILLO Adriana; \& FLÓREZ, María del Pilar (2009). Los niveles de comprensión lectora: hacia una enunciación investigativa y reflexiva para mejorar la comprensión lectora en estudiantes universitarios. Actualidades Pedagógicas, 53, 95-107. https://ciencia.lasalle. edu.co/ap/vol1/iss53/8/

GRAESSER, Arthur; LEÓN, José; \& OTERO José (2002). Introduction to the psychology of science text comprehension. En José Otero; José A. León; \& Arthur C. Graesser (Eds.), The psychology of science text comprehension (pp. 1-15). Lawrence Erlbaum Associates.

GRAESSER, Arthur; MILLIS, Keith; \& ZWAAN, Rolf (1997). Discourse comprehension. Annual Review of Psychology, 48, 163-189. https://doi.org/10.1146/annurev.psych.48.1.163

HERNÁNDEZ, Gerardo (1998). Paradigmas en psicología de la educación. Paidós.

HERNÁNDEZ-GARRE, Carmen María; \& AVILÉS-SOLER, Borja (2015). E.S.C.L.O.S.A.: programa de orientación como ayuda al alumnado universitario en el EEES. Revista de Estudios e Investigación en Psicología y Educación, (3), 11-014. https://doi.org/10.17979/reipe. 2015.0.03.175 
JIMÉNEZ, Lourdes; BARIDON, Daniela; \& MANZANAL, Ana Isabel (2016). Análisis del control de la comprensión lectora en textos científicos en alumnos de Secundaria Obligatoria y Bachillerato. Revista Complutense de Educación, 27(1), 285-307. https://doi.org/10.5209/ rev_RCED.2016.v27.n1.45749

KINTSCH, Walter; \& VAN DIJK, Teun, A. (1978). Toward a Model of Text Comprehension and Production. Psychological Review, 85(5), 363-394. https://doi.org/10.1037/0033-295X. 85.5 .363

LÓPEZ-BONILLA, Guadalupe (2017). Alfabetización y literacidad disciplinar: el acceso al conocimiento en las disciplinas académicas. En Elsa Margarita Ramírez-Leyva (Coord.), La enseñanza de la lectura en la universidad (pp. 29-42). UNAM. https://doi.org/10.22201/ iibi.9786070291814e.2017

LÓPEZ-DELGADO, María de la Luz (2002). Estrategias de comprensión. [Tesis de doctorado, Universidad de Castilla La Mancha]. Repositorio Institucional de la Universidad de Castilla La Mancha. https://ruidera.uclm.es/xmlui/handle/10578/926

MATEOS, Mar (2009). Aprender a leer textos académicos: Más allá de la lectura reproductiva. En Juan Ignacio Pozo; \& M. del Puy Pérez-Echeverría (Coords.), Psicología del aprendizaje universitario: La formación en competencias (pp.106-119). Morata.

MATURANO, Carla Inés; SOLIVERES, María Amalia; \& MACÍAS, Ascensión. (2002). Estrategias cognitivas y metacognitivas en la comprensión de un texto de ciencias. Enseñanza de las ciencias, 20(3), 415-425. https://www.raco.cat/index.php/Ensenanza/article/view/21831

MOKHTARI, Kouider; \& REICHARD, Carla (2002). Assessing Students' Metacognitive Awareness of Reading Strategies. Journal of Educational Psychology, 94(2), 249-259. https://doi.org/ 10.1037/0022-0663.94.2.249

MOMSEN, Jennifer; LONG, Tammy; WYSE, Sara; \& EBERT-MAY, Diane (2010). Just the facts? Introductory undergraduate biology courses focus on low-level cognitive skills. CBE. Life Sciences Education, 9(4), 435-440. https://doi.org/10.1187/cbe.10-01-0001

MONEREO, Carles (Coord.). (1999). Estrategias de enseñanza y aprendizaje. Formación del profesorado y aplicación en la escuela. (6a. ed.). Graó.

PÉREZ, Gastón; \& GONZÁLEZ-GALLI, Leonardo Martín (2020). Una posible definición de metacognición para la enseñanza de ciencias. Investigações em Ensino de Ciências, 25(1), 384-404. https://doi.org/10.22600/1518-8795.ienci2020v25n1p384

PÉREZ-ZORRILLA, Mã Jesús (2005). Evaluación de la comprensión lectora: dificultades y limitaciones. Revista de Educación, num. Extraordinario, 121-138. http://www.educacion 
yfp.gob.es/revista-de-educacion/numeros-revista-educacion/numerosanteriores/2005/re2005/re2005-08.html

PERETZ, Arna (1986). Do content area passages affect student performance on reading comprehension tests? Paper presented at a 20th Meeting of the International Association of Teachers of English as a Foreign Language (Brighton, England, April 1-4, 1986), 19 pp.

UGARRIZA, Nelly (2006). La comprensión lectora inferencial de textos especializados y el rendimiento académico de los estudiantes universitarios del primer ciclo. Persona, 9, 3176. https://doi.org/10.26439/persona2006.n009.902

VALENZUELA, Ángel (2018). La metacognición en los procesos de lectura y escritura académica: ¿qué nos dice la literatura? Lenguaje 46(1), 69-93. https://doi.org/10.25100/lenguaje. v46i1.6197

VAN DIJK, Teun A. (1993). Modelos en la Memoria. El papel de las representaciones de la situación en el procesamiento del discurso. Revista Latina de Pensamiento y Lenguaje, 2(1), 39-55.

VAN DIJK, Teun A.; \& KINTSCH, Walter (1983). Strategies of Discourse Comprehension. Academic Press.

Fecha de recepción: 2 de noviembre de 2020. Fecha de revisión: 27 de marzo de 2021. Fecha de aceptación: 29 de marzo de 2021. Fecha de publicación: 1 de julio de 2021. 